\title{
Pregnancy outcomes in patients with tubal infertility following laparoscopic treatment: a multivariate predictive study
}

\author{
Liyi Zhang, Fengxi Jiang, Ruihong Qi, Wei Fu, Yuzhen Wang, Dingqing Gui \\ Department of Gynaecology, Dazhou Central Hospital, Dazhou, China \\ Contributions: (I) Conception and design: L Zhang, D Gui; (II) Administrative support: F Jiang; (III) Provision of study materials or patients: L \\ Zhang, F Jiang, R Qi, W Fu; (IV) Collection and assembly of data: All authors; (V) Data analysis and interpretation: L Zhang, R Qi, W Fu, Y Wang, \\ D Gui; (VI) Manuscript writing: All authors; (VII) Final approval of manuscript: All authors. \\ Correspondence to: Dingqing Gui. Department of Gynaecology of Dazhou Central Hospital, Dazhou 635000, China. Email: zly29225079@163.com.
}

Backgroundr Tubal infertility represents a large portion of female infertility. This study analyzed the pregnancy outcomes of patients with tubal infertility after laparoscopic treatment. A multivariate predictive analysis was also conducted.

Methods: The clinical data of 92 patients admitted in our hospital from March 2015 to March 2018 with tubal infertility were analyzed. According to the inclusion and exclusion criteria, 87 patients were finally included, and all patients were treated with laparoscopy. The clinical data of all study subjects were collected, including age, years of infertility, type of infertility, history of pelvic surgery, history of tubal pregnancy, history of artificial abortion, and lowest tubal function score. The patients were followed up for two years, and multiple logistic regression was used to analyze the factors affecting the pregnancy outcomes of patients with tubal infertility after laparoscopic treatment. The receiver operating characteristic (ROC) curve was used to analyze the predictive values.

Results: Among the 87 patients, 63 cases (72.41\%) had successful pregnancies at follow-up, and 24 cases (27.58\%) were not pregnant. The time of follow up (half a year, 1 year, and 2 years) was not significantly different between the pregnant and the non-pregnant groups. There were no significant differences in the infertility types, pelvic surgery history, and induced abortion history between two groups $(\mathrm{P}>0.05)$, However, there were differences in the age, years of infertility, tubal pregnancy history, and lowest tubal function score $(\mathrm{P}<0.05)$. Multivariate analyses showed that the patient's age over 35 years, a lowest tubal function score indicating severe injury, and a history of tubal pregnancy were independent risk factors for the pregnancy outcome of patients with tubal infertility after laparoscopic treatment $(\mathrm{P}<0.05)$. The area under the ROC curve for age, lowest tubal function score, tubal pregnancy history, and the three combined curves were 0.792 , $0.852,0.816$, and 0.949 , respectively.

Conclusions: The age of the patient, the lowest tubal function score, and the tubal pregnancy history are independent risk factors for the pregnancy outcome of patients with tubal infertility after laparoscopic treatment. Furthermore, the combination of the three risk factors can be used as a predictor of the pregnancy outcome in patients with tubal infertility after laparoscopic treatment.

Keywords: Tubal infertility; laparoscopic treatment; pregnancy outcome; multivariate logistic regression analysis

Submitted Nov 11, 2020. Accepted for publication Dec 29, 2020.

doi: 10.21037/apm-20-2347

View this article at: http://dx.doi.org/10.21037/apm-20-2347 


\section{Introduction}

Tubal infertility accounts for $36 \%$ of all female infertilities (1). Blockage or dysfunction of the fallopian tubes is the main cause of tubal infertility, with proximal tubal blockage accounting for about $26 \%$ of all infertilities. Blockage is commonly caused by acute or chronic inflammation of the fallopian tubes. The inflammation can infiltrate the inner membrane of the fallopian tube, impairing its function and the ability of the sperm and egg to combine, thereby resulting in infertility (2). In addition, severe inflammation in the fallopian tubes can cause fallopian tube edema and mucosal cell shedding, tubal effusion, and aggravate clinical symptoms and infertility symptoms (3). At present, the clinical treatment of patients with tubal infertility is mainly based on fluid drainage. However, related studies have found limitations to this method as tubal patency is subjectively assessed by the degree of pain felt by the patient. In addition, the rate of tubal patency is nearly always unsatisfactory. Alternatively, laparoscopic treatment may be beneficial as it improves the efficacy of dredging and reduces the occurrence of adverse reactions (4). Therefore, a multivariate predictive study was conducted on patients with tubal infertility who were treated with laparoscopy, and their pregnancy outcomes were analyzed. We present the following article in accordance with the STARD reporting checklist (available at http://dx.doi.org/10.21037/apm-20-2347).

\section{Methods}

\section{General information}

The clinical data of 92 patients with tubal infertility admitted to our hospital from March 2015 to March 2018 were analyzed. The inclusion criteria were as follows: (I) non-pregnant adult females who had been married for more than one year with a normal sex life and no contraceptive measures; (II) patients who were diagnosed with fallopian tube disease by laboratory and other related tests; (III) patients with no surgical contraindications; and (IV) patients with regular postoperative sexual activity and no contraceptive measures. The exclusion criteria were as follows: (I) infertility caused by other dysfunctions such as male oligospermia and impotence; (II) irregular or infrequent sexual activity; (III) a history of tubal ligation surgery; (IV) patients with immunodeficiencies; and (V) patients who cannot cooperate due to mental disorders; (VI) patients indicated by laparoscopy for female infertility caused by other reasons Based on the inclusion and exclusion criteria, a total of 87 patients aged between 24 to 41 years (average $32.47 \pm 4.29$ years), with an infertility period of 3 to 5 years (average $3.15 \pm 1.38$ years) were included in this study. This study was approved by the Dazhou Central Hospital (No. 2019029) and was conducted in accordance with the Declaration of Helsinki (as revised in 2013). Individual consent for this retrospective analysis was waived.

\section{Surgical methods}

All patients underwent routine checks including blood, leucorrhea, electrocardiogram, coagulation, and other related indicators before surgery. Sexual activity was prohibited three days prior to surgery.

The laparoscopic instrument used in this study was from Stryker Corporation (State of Michigan, USA). The patients were administered general anesthesia, and placed in the lithotomy position with their heads kept low and the buttocks kept high. The classic three-hole technique was used to enter the abdomen. After entering the abdominal cavity, the pelvic cavity was examined, and the fallopian tube was opened and separated or shaped depending on the specific condition of the fallopian tube. After the surgery, the fallopian tube was treated with fluid drainage, and the degree of obstruction was visualized under the microscope. If the proximal end of both fallopian tubes was completely blocked during the surgery, but the distal end of the fallopian tube was unobstructed with intact tissue structure, the treatment was adjusted to laparotomy for uterine tube implantation.

After the surgery, the patients were followed up for 2 years, through on-site visits, telephone consults, and other methods. The outcomes included normal pregnancy, ectopic pregnancy, and miscarriage.

\section{Observation indicators}

General patient information was collected, including age, years of infertility, type of infertility, history of pelvic surgery, history of tubal pregnancy, history of induced abortion, and the lowest tubal function score. The lowest tubal function score was performed by an experienced chief physician in the undergraduate section. The sum of the scores of the bilateral fallopian tubes is used as a measure of the degree of injury, with 7-8 points classified as mild injury, 4-6 points classified as moderate injury, and $0-3$ 
Table 1 Pregnancy outcomes of tubal infertility patients at half a year, one year, or two years after laparoscopic treatment

\begin{tabular}{lccc}
\hline Period & Normal pregnancy & Ectopic pregnancy & Not pregnancy \\
\hline Half a year & $19(21.83)$ & $5(5.74)$ & $7(8.04)$ \\
1 year & $17(19.54)^{\mathrm{a}}$ & $4(4.59)^{\mathrm{a}}$ & $9(10.34)^{\mathrm{a}}$ \\
2 years & $14(16.09)^{\mathrm{ab}}$ & $4(4.59)^{\mathrm{ab}}$ & $8(9.19)^{\mathrm{ab}}$ \\
\hline
\end{tabular}

${ }^{a}, P>0.05$ was compared with half a year after treatment; ${ }^{b}, P>0.05$ was compared with one year after treatment. The data in brackets were shown as composition ratio.

points classified as severe injury.

\section{Statistical methods}

The data in this study were statistically analyzed using SPSS 22.0 software (IBM, New York, USA). Multiple logistic regression was used to analyze the factors affecting the patient's pregnancy outcome. The measurement data were presented as the mean \pm standard deviation $\left(\bar{x}_{ \pm}\right)$, and analyzed using the F/t test. The count data was described as rate or composition ratio and analyzed using the $\chi^{2}$ test. The receiver operating characteristic (ROC) curve was drawn to analyze the predictive value of laparoscopic treatment of pregnancy infertility in patients with tubal infertility. Results with $\mathrm{P}<0.05$ were considered statistically significant.

\section{Results}

The pregnancy outcome of patients with tubal infertility at half a year, 1 year or 2 years after laparoscopic treatment

Following laparoscopic treatment on the 87 patients, a total of $63(72.41 \%)$ achieved a normal pregnancy and $24(27.58 \%)$ were not pregnant. However, there was no significant difference between the groups at half a year, 1 year, or 2 years after the surgery $(\mathrm{P}>0.05$, Table 1$)$.

\section{Single factor analysis of the pregnancy outcome in patients with tubal infertility after laparoscopic treatment}

The results of single factor analyses demonstrated that there were significant differences in the age, years of infertility, tubal pregnancy history, and lowest tubal function score between the pregnant and the non-pregnant groups $(\mathrm{P}<0.05)$. However, no differences were observed in the two groups when comparing infertility, pelvic surgery history, and induced abortion $(\mathrm{P}>0.05$, Table 2).

\section{Multivariate analysis of factors affecting pregnancy outcome of patients with tubal infertility after laparoscopic treatment}

The results of multivariate analyses demonstrated that factors such as the patient's age greater than 35 years, a lowest tubal function score showing severe injury, and a history of tubal pregnancy were all independent risk factors for the pregnancy outcome of patients with tubal infertility after laparoscopic treatment $(\mathrm{P}<0.05$, Table 3).

\section{The predictive value of pregnancy outcomes in patients with tubal infertility after laparoscopic treatment}

The ROC curve was used to analyze the predictive value of age, the lowest tubal function score, the history of tubal pregnancy, and the combination of the three factors in patients with tubal infertility following laparoscopic treatment. The area under the curve of age, tubal lowest functional score, history of tubal pregnancy, and the combination of the three were $0.792,0.852,0.816,0.949$, respectively, indicating that the area under the curve of the combined factors was the largest (Table 4 and Figure 1).

\section{Discussion}

In recent years, with the increased incidences of reproductive tract infections in women of gestational age in China, the prevalence of tubal infertility has also escalated significantly (5). According to epidemiological statistics, tubal infertility is a common type of clinical infertility and accounts for a third of all causes of infertility, and as such, seriously affects the health and quality of life of patients (6). The traditional methods of diagnosis and treatment for infertility are iodine hydrography of uterine salpinx, Chinese and Western medicine treatment and physical therapy. The laparotomy is difficult to be accepted by patients for the large injury after treatment, the unsure 
Table 2 Single factor analyses of the pregnancy outcomes in patients with tubal infertility after laparoscopic treatment

\begin{tabular}{|c|c|c|c|c|}
\hline Factor & $\begin{array}{l}\text { Pregnancy } \\
\quad(n=63)\end{array}$ & $\begin{array}{l}\text { Infertility } \\
(n=24)\end{array}$ & $\chi^{2}$ & $P$ \\
\hline Age (years) & & & 18.202 & 0.000 \\
\hline$\leq 35$ & 45 & 5 & & \\
\hline$>35$ & 18 & 19 & & \\
\hline Infertility time (years) & & & 11.240 & 0.001 \\
\hline$\geq 3$ & 22 & 18 & & \\
\hline$<3$ & 41 & 6 & & \\
\hline Infertility type & & & 0.022 & 0.881 \\
\hline Primary & 33 & 13 & & \\
\hline Secondary & 30 & 11 & & \\
\hline Pelvic surgery history & & & 0.004 & 0.947 \\
\hline Yes & 32 & 12 & & \\
\hline No & 31 & 12 & & \\
\hline Tubal pregnancy history & & & 19.344 & 0.000 \\
\hline Yes & 15 & 18 & & \\
\hline No & 48 & 6 & & \\
\hline Induced abortion history & & & 0.000 & 0.987 \\
\hline Yes & 29 & 11 & & \\
\hline No & 34 & 13 & & \\
\hline $\begin{array}{l}\text { Lowest tubal function } \\
\text { score }\end{array}$ & & & 23.937 & 0.000 \\
\hline Mild to moderate injury & 49 & 5 & & \\
\hline Severe injury & 14 & 19 & & \\
\hline
\end{tabular}

efficacy, and there are false negative hysterosalpingography or asymptomatic patients with subclinical lesions, which is often neglect by the patient or the doctor with the threaten of delay of treatment. Therefore, it is imperative that tubal infertility is treated effectively so as to improve patient pregnancy outcomes (7).

With the development of laparoscopic technology and the improvement of surgical techniques, laparoscopy has become widely used in clinical practice. Laparoscopic surgery is currently the preferred method of clinical treatment in tubal infertility patients (8). Laparoscopy allows for the clear visualization of the obstruction and stenosis of the fallopian tubes, effective observation of the pelvic condition, correct evaluation of the shape and function of the fallopian tube, and direct visualization of the relationship between the fallopian tube and the surrounding tissues. Therefore, it allows the clinician to clearly diagnose pelvic diseases and assess the status of the fallopian tubes for particular treatment. Laparoscopic treatment has been shown to be the most effective method of restoring the natural physiological state and function of the fallopian tubes (9). Laparoscopy has the advantages of less trauma, faster recovery, and reduced postoperative complications (10). Relevant studies have shown that laparoscopy can comprehensively assess the functional status of the patient's fallopian tubes and deliver targeted treatment, which is beneficial to increasing pregnancy rates and improving pregnancy outcomes (11). Wang (12) studied 181 patients with tubal infertility and showed that the normal pregnancy rate was as high as $61.33 \%$ at three years after laparoscopic surgery of the fallopian

Table 3 Multivariate analyses of the factors influencing pregnancy outcome after laparoscopic treatment of tubal infertility

\begin{tabular}{|c|c|c|c|c|c|}
\hline Factor & Regression coefficient & SEM & Wald $\chi^{2}$ & $P$ & OR $(95 \% \mathrm{Cl})$ \\
\hline Age (>35 vs. $\leq 35$ years) & 0.869 & 0.161 & 4.596 & $<0.001$ & $2.38(1.74-3.26)$ \\
\hline $\begin{array}{l}\text { Lowest tubal function score (mild to } \\
\text { moderate injury vs. severe injury) }\end{array}$ & 0.893 & 0.325 & 4.523 & $<0.001$ & $2.44(1.29-4.62)$ \\
\hline
\end{tabular}

SEM, standard error of mean; OR, odd ratio; Cl, confidence interval. 
Table 4 Predictive value of factors on the pregnancy outcome after laparoscopic treatment in patients with tubal infertility

\begin{tabular}{lccccc}
\hline Predictive factor & Optimal threshold & Sensitivity & Specificity & AUC of ROC & 95\% Cl \\
\hline Age & 25.64 & 0.742 & 0.763 & 0.792 & $0.614-0.969$ \\
Tubal pregnancy history & 34.42 & 0.765 & 7.754 & 0.852 & $0.711-0.993$ \\
Lowest tubal function score & 36.41 & 0.759 & 0.748 & 0.816 & $0.666-0.966$ \\
Triadic combined test & 37.45 & 0.864 & 0.886 & 0.789 & 0.768 \\
Predictive exponential model & 1.687 & 0.354 & $0.754-0.865$ \\
\hline
\end{tabular}

AUC, area under the curve; ROC, receiver operating characteristic curve; $\mathrm{Cl}$, confidence interval.

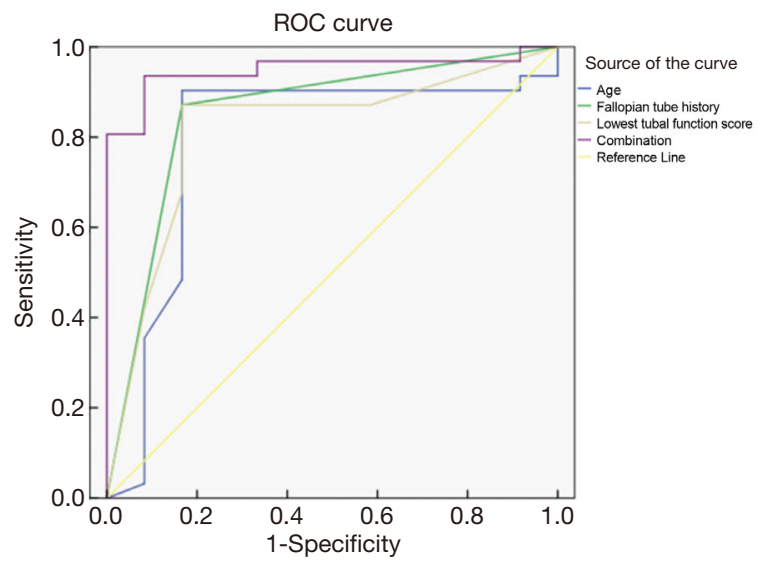

Figure 1 The predictive value of factors on the pregnancy outcome after laparoscopic treatment in patients with tubal infertility.

tubes. In this current study, the pregnancy rate of patients with tubal infertility after laparoscopic treatment was $72.41 \%$, indicating that the clinical efficacy of laparoscopic treatment in patients with tubal infertility was sufficient to achieve an ideal postoperative pregnancy rate. These results support that notion that laparoscopic surgery is a beneficial clinical treatment for patients with tubal infertility (13).

To verify the impact of laparoscopic treatment on tubal infertility patients, this study conducted a 2-year follow-up for the 87 patients. The results demonstrated that during the follow-up period, 24 patients did not fall pregnant, and the infertility rate was $27.58 \%$. Previous research has shown that the clinical efficacy of laparoscopic treatment on tubal infertility is directly related to the patient's age, tubal function score, history of miscarriage, and other factors (14). Related studies have also found that for patients with tubal infertility treated with laparoscopic methods, tubal function scores, pelvic and tubal lesion locations and degrees, and years of infertility are risk factors for poor pregnancy $(15,16)$.
In agreement with the above reports, this study revealed that the patient's age greater than 35 years, a lowest tubal function score indicating severe injury, and a history of tubal pregnancy were independent risk factors for poor pregnancy outcomes in patients with tubal infertility after laparoscopic treatment $(\mathrm{P}<0.05)$. This study further used the ROC curves to analyze the predictive value of age, the lowest tubal function score, the tubal pregnancy history, and the combination of the three factors in the prognosis of patients with tubal infertility after laparoscopic treatment. The results showed that the combination of the three factors had the largest area under the curve, and hence, the combined test achieved the highest predictive value. Therefore, the combined evaluation of the three factors can be used in the prediction of pregnancy outcomes for patients with tubal infertility after laparoscopic treatment.

Therefore, for patients over 35 years of age, active intervention measures should be considered based on the specific condition of the patient, and surgical intervention should be carried out as soon as possible to improve the therapeutic benefits. A large number of clinical manifestations have shown that for patients with a history of tubal pregnancy and a lowest tubal function score indicating severe injury, the postoperative pregnancy rate is decreased (17). In fact, the lowest tubal function score system is often used as a reference for gynecologists to predict postoperative pregnancy rates. The lower the score, the lower the postoperative pregnancy rate (18). Therefore, depending on comprehensive factors such as the actual situation of the patient and the patient's economic status, in vitro fertilization embryo transfer and other interventionassisted reproduction treatments may be considered to improve the pregnancy rate of these patients, so as not to miss the best time for treatment of patients (19). Due to the small sample size of this study, the bias of the experimental results was inevitable. Therefore, the sample size should 
be expanded in further research to obtain more accurate experimental results.

In summary, the patient's age, the lowest tubal function score, and the tubal pregnancy history are independent risk factors for the pregnancy outcome of patients with tubal infertility after laparoscopic treatment. The combination of these three risk factors can be used as a predictive index to evaluate the outcome of pregnancy in such patients.

\section{Acknowledgments}

Funding: None.

\section{Footnote}

Reporting Checklist: The authors have completed the STARD reporting checklist. Available at http://dx.doi.org/10.21037/ apm-20-2347

Data Sharing Statement: Available at http://dx.doi. org/10.21037/apm-20-2347

Conflicts of Interest: All authors have completed the ICMJE uniform disclosure form (available at http://dx.doi. org/10.21037/apm-20-2347). The authors have no conflicts of interest to declare.

Ethical Statement: The authors are accountable for all aspects of the work in ensuring that questions related to the accuracy or integrity of any part of the work are appropriately investigated and resolved. This study was approved by the Dazhou Central Hospital (No. 2019029) and was conducted in accordance with the Declaration of Helsinki (as revised in 2013). Individual consent for this retrospective analysis was waived.

Open Access Statement: This is an Open Access article distributed in accordance with the Creative Commons Attribution-NonCommercial-NoDerivs 4.0 International License (CC BY-NC-ND 4.0), which permits the noncommercial replication and distribution of the article with the strict proviso that no changes or edits are made and the original work is properly cited (including links to both the formal publication through the relevant DOI and the license). See: https://creativecommons.org/licenses/by-nc-nd/4.0/.

\section{References}

1. Sun F, Yang S, Yang Y, et al. Laparoscopic Management of 42 Cases of Tubal Stump Pregnancy and Postoperative Reproductive Outcomes. J Minim Invasive Gynecol 2020;27:618-24.

2. Li JY. Clinical value of hysteroscopic and laparoscopic surgery in the treatment of tubal obstructive infertility. China Medicine and Pharmacy 2019;21:156-8.

3. Wu H, Wang ZH, Zhou LQ. Effect of GnRH-a after laparoscopic surgery on pregnancy outcome of EMT patients with infertility. Modern Diagnosis and Treatment 2019;31:542-3.

4. Yuan L, Jingying $\mathrm{H}$, Xiujuan $\mathrm{C}$, et al. Predictive value of a modified classification of fallopian tube status on prognosis of tubal factor infertility after laparoscopic surgery. Medicine (Baltimore) 2019;98:e14952.

5. Philipps W, Fietz AK, Meixner K, et al. Pregnancy outcome after first-trimester exposure to fosfomycin for the treatment of urinary tract infection: an observational cohort study. Infection 2020;48:57-64.

6. Gupta RA, Pokharia P, Khanduri A. Laparoscopic treatment of duplicate gallbladder with acute cholecystitis. J Minim Access Surg 2020;16:447-8.

7. Domali E, Mantzioros R, Kathopoulis N, et al. P14.04: Laparoscopic treatment of ectopic tubal pregnancy: an old fashion approach? Ultrasound Obstet Gynecol 2019;54:199-200.

8. Amimoto S, Kurita T, Mori H, et al. Naturally conceived ipsilateral interstitial pregnancy after salpingectomy due to tubal pregnancy. Japanese Journal of Gynecologic and Obstetric Endoscopy 2019;24:178-9.

9. Nishida M, Miyamoto Y, Kawano Y, et al. A case of successful laparoscopic surgery for tubal stump pregnancy after tubectomy. Clin Med Insights Case Rep 2015;8:1-4.

10. Lafalla O, Esteban LM, Lou AC, et al. Clinical utility of thrombophilia, anticoagulant treatment, and maternal variables as predictors of placenta-mediated pregnancy complications: an extensive analysis. J Matern Fetal Neonatal Med 2021;34:588-98.

11. Karpishchenko SA, Sopko ON, Bervinova AN. Conservative treatment of exacerbation of chronic purulent otitis media. Vestn Otorinolaringol 2020;85:41-4.

12. Wang JC. Effect of heat-sensitive moxibustion combined with acupoint injection on pregnancy outcome after 
hysteroscopic and laparoscopic surgery for hydrosalpinx infertility. China Modern Doctor 2019;25:347-8.

13. Fan XD, Wang Y, Zhu YJ. Analysis of the Feasibility and Outcome of Laparoscopic Treatment of Hetertopic Pregnancy. Journal of International Obstetrics and Gynecology 2019;23:167-8.

14. Wang KL, Qi XJ. Analysis of influence factors of pregnancy outcome in patients with ovarian endometriomas after laparoscopic surgery. J Reprod Med 2019;52:431-3.

15. Coelho JCU, Hosni AVE, Claus CM, et al. Treatment of median arcuate ligament syndrome: outcome of laparoscopic approach. Arq Bras Cir Dig 2020;33:e1495.

16. Duan L, Zhang YY, Wang YX. Evaluation Value of 4D Contrast-enhanced Ultrasonography in Tubal Patency after Tubal Pregnancy Treatment. World Latest Medicine
Information 2019;15:342-3.

17. Chen F, Zhang Y, Cheng JM. Analysis of Pregnancy Outcome of Moderate and Severe Intrauterine Adhesions after Transcervical Resection of Intrauterine Adhesions. Journal of International Obstetrics and Gynecology 2019;26:350-2.

18. Ding J, Shao JH, Yang L. Clinical study of hysteroscopylaparoscopy combined with assisted reproductive technology in the treatment of tubal infertility. Shanghai Medical \& Pharmaceutical Journal 2019;16:153-4.

19. Lei X, Liu J, Yu GM. Analysis on influencing factors of pregnancy rate after laparoscopic surgery in patients with hydrosalpinx. Journal of Minimally Invasive Medicine 2019;13:172-3.

(English Language Editor: J. Teoh)
Cite this article as: Zhang L, Jiang F, Qi R, Fu W, Wang Y, Gui D. Pregnancy outcomes in patients with tubal infertility following laparoscopic treatment: a multivariate predictive study. Ann Palliat Med 2021;10(1):462-468. doi: 10.21037/apm20-2347 